\title{
Quantitative adsorbate structure determination under catalytic reaction conditions
}

\author{
D. Kreikemeyer-Lorenzo, ${ }^{1}$ W. Unterberger, ${ }^{1}$ R. Blume, ${ }^{1}$ M. Hävecker, ${ }^{1}$ T. C. R. Rocha, ${ }^{1}$ A. Knop-Gericke, ${ }^{1}$ R. Schlögl, ${ }^{1}$ \\ T. J. Lerotholi, ${ }^{2, *}$ D. A. Duncan, ${ }^{2}$ and D. P. Woodruff ${ }^{2, \dagger}$ \\ ${ }^{1}$ Fritz-Haber-Institut der Max-Planck-Gesellschaft, Faradayweg 4-6, 14195, Berlin, Germany \\ ${ }^{2}$ Physics Department, University of Warwick, Coventry CV4 7AL, United Kingdom
}

(Received 16 November 2012; revised manuscript received 13 February 2013; published 19 March 2013)

\begin{abstract}
Current methods allow quantitative local structure determination of adsorbate geometries on surfaces in ultrahigh vacuum (UHV) but are incompatible with the higher pressures required for a steady-state catalytic reactions. Here we show that photoelectron diffraction can be used to determine the structure of the methoxy and formate reaction intermediates during the steady-state oxidation of methanol over $\mathrm{Cu}(110)$ by taking advantage of recent instrumental developments to allow near-ambient pressure x-ray photoelectron spectroscopy. The local methoxy site differs from that under static UHV conditions, attributed to the increased surface mobility and dynamic nature of the surface under reaction conditions.
\end{abstract}

DOI: 10.1103/PhysRevB.87.125420

PACS number(s): 68.43.Fg, 68.49.Jk, 82.65.+r

Traditional surface science studies under ultrahigh vacuum (UHV) conditions that allow the use of the full armory of electron, photon, and ion probes have achieved enormous advances in our understanding of the structural, electronic, and chemical properties of well-characterized surfaces in the last few decades. There is, however, an increasing recognition that the huge differences in pressure of reactant gases from UHV $\left(\sim 10^{-13}\right.$ atmospheres) to atmospheric pressure and above, which characterize practical processes in heterogeneous catalysis and corrosion, can lead to fundamental differences in the dominant physics and chemistry. Moreover, higher pressures are essential to allow in situ or in operando studies of the species present on a surface during a catalytic reaction; however, in UHV, only a static state, often corresponding to a nominal equilibrium, can be investigated. A number of techniques, therefore, are being developed to address this pressure gap. Optical spectroscopies are well known to bridge this gap both on dispersed catalysts (e.g., Refs. 1 and 2) and on single crystal surfaces (e.g., Ref. 3), and recent progress has also been made on the use of scanning tunneling microscopy (STM) to surface reactions. ${ }^{4}$ Moreover, early attempts to extend the pressure range of x-ray photoelectron spectroscopy (XPS) ${ }^{5}$ using differential pumping have recently been advanced using synchrotron radiation, and these near-ambient pressure XPS (NAP-XPS) studies, while still under low vacuum conditions, have proved to be very fruitful. ${ }^{6-10}$ These intermediate pressures, covering the wide range of $\sim 10^{-6}-10 \mathrm{mbar}$, are high enough to allow in situ studies of the surface species, which are present during some steady-state catalytic reactions to be monitored provided that their coverage under these conditions is sufficient, although the accessible pressures fall short of those required for true in operando studies. The use of novel electron-transparent window materials can also allow studies of liquids (but not solid surfaces exposed to gases) at ambient pressure. ${ }^{11}$

Quantitative experimental structural information, however, is needed to understand reaction mechanisms. Increasingly sophisticated theoretical treatments are proving extremely successful in modeling these surface processes (e.g., Ref. 12), but complementary experimental information is essential to refine these methods. Surface x-ray diffraction (SXRD) (e.g., Refs. 13 and 14) can address this problem, but it relies on long-range order that is often absent for surface species under reaction conditions. Here we show that a local quantitative structural technique that is element- and chemicalstate specific, namely scanned-energy mode photoelectron diffraction $(\mathrm{PhD})$, can be used to determine the adsorption geometry of a molecular surface reaction intermediate during a steady-state catalytic reaction. Specifically, we determine the local structure of the key surface intermediate of the methanol oxidation reaction to produce formaldehyde over $\mathrm{Cu}(110)$. We show the local geometry of this deprotonated methanol or methoxy species, $\mathrm{CH}_{3} \mathrm{O}$-, is subtly different from that found in the well-ordered $\mathrm{Cu}(110)(5 \times 2)$-methoxy phase formed under static UHV conditions. We attribute this difference to the distinct surface dynamics associated with the steady-state reaction at higher chemical potentials provided by the $10^{-5}$ mbar pressure and elevated temperature.

The $\mathrm{PhD}$ technique ${ }^{15,16}$ involves the measurement of the core-level photoelectron intensity in fixed directions as a function of photon energy and, hence, photoelectron energy. Modulations in this intensity occur because of coherent interference of the directly emitted component of the photoelectron wavefield and other components elastically scattered by surrounding (mainly substrate) atoms. As the photoelectron energy and its associated wavelength varies, these scattering paths switch in and out of phase, and analysis of the resulting modulations in the photoelectron intensity allows the local emitter-scatterer geometry to be determined. In the present case, in which both methoxy and formate bond to the surface through the $\mathrm{O}$ atoms, $\mathrm{PhD}$ measurements from the $\mathrm{O} 1 \mathrm{~s}$ emission are particularly sensitive to the local adsorption geometry because of the dominant role of nearest-neighbor $\mathrm{Cu}$ backscattering. By exploiting the recent developments in NAP-XPS, we show that the PhD technique can be extended to higher pressures.

The experiments were performed at the beamline Innovative Station for In Situ Spectroscopy (ISISS) installed on the BESSY II synchrotron radiation source of the Helmholtz Zentrum Berlin, ${ }^{17}$ equipped with a NAP-XPS instrument, a gas-dosing system, and a mass spectrometer to monitor the surface reaction products. The monochromated soft $\mathrm{x}$-ray beam from BESSY enters the chamber through a $\operatorname{SiN}_{x}$ window at an incidence angle of $55^{\circ}$ relative to the surface 
normal along which the photoemission is measured by a hemispherical electron energy analyzer. The $\mathrm{Cu}(110)$ sample was cleaned in situ by several cycles of $1.5 \mathrm{keV}$ argon ion bombardment and annealing to $\sim 800 \mathrm{~K}$, together with oxygen treatment to remove residual carbon. XPS and PhD measurements were made while the surface was exposed to a mixture of methanol and oxygen with a ratio of $3: 2$ at total pressures mainly in the $10^{-5}$ mbar range. $\mathrm{O} 1 \mathrm{~s} \mathrm{PhD}$ modulation spectra were obtained by measuring photoelectron energy distribution curves (EDCs) of the $\mathrm{O} 1 \mathrm{~s}$ peaks, at $4 \mathrm{eV}$ steps in photon energy, over the photoelectron kinetic energy range of 50-350 eV. These data were processed following our general $\mathrm{PhD}$ methodology (e.g., Refs. 15 and 16), in which the individual EDCs are fitted by Gaussian peaks for each chemically shifted component, a Gauss error function (step), and a template background. The final $\mathrm{PhD}$ modulation spectra obtained from the integrated area of each peak as a function of photoelectron kinetic energy, $I(E)$, are given by $\chi(E)=\left(I(E)-I_{0}(E)\right) / I_{0}(E)$, where $I_{0}(E)$, representing the nondiffractive intensity (including instrumentally introduced variations), is obtained from a smooth spline through $I(E)$.

The catalytic reaction of methanol with oxygen over $\mathrm{Cu}(110)$ has been studied extensively in the past by temperature-programmed reaction spectroscopy (e.g., Refs. 18-21) and direct measurements of the reaction turnover frequency, ${ }^{22}$ while electronic and vibrational spectroscopies (e.g., Refs. 19 and 22-24) provided direct identification of the surface reaction intermediates. The consensus is that the key molecular surface intermediate is the methoxy species, which produces the desired products of formaldehyde $\left(\mathrm{CH}_{2} \mathrm{O}\right)$ and hydrogen after further reaction. An alternative reaction pathway that leads to the undesired total combustion (producing $\mathrm{CO}_{2}$ and $\mathrm{H}_{2} \mathrm{O}$ ) occurs via the formate ( $\mathrm{HCOO}$ ) surface intermediate, which can be created from interaction of methoxy with excess surface oxygen.

The local structures of both the methoxy and formate species on $\mathrm{Cu}(110)$ have been determined under static UHV conditions at $\sim 140 \mathrm{~K}$ using the $\mathrm{PhD}$ technique. Methoxy is found to bond at short-bridge sites through the $\mathrm{O}$ atom (midway between two nearest-neighbor $\mathrm{Cu}$ atoms in the close-packed [110] azimuth), partly on $\mathrm{Cu}$ adatoms and partly on the underlying surface (Fig. 1), with $\mathrm{Cu}-\mathrm{O}$ bondlengths of $1.98 \pm$ $0.03 \AA$ and $1.90 \pm 0.03 \AA$, respectively. ${ }^{25}$ Formate bonds symmetrically through its two oxygen atoms, centered on a bridging site with its molecular plane perpendicular to the surface, such that the two $\mathrm{O}$ atoms are close to atop $\mathrm{Cu}$ surface atoms (Fig. 1). ${ }^{26-28}$ NAP-XPS has identified the presence of both methoxy and formate on the surface under methanol oxidation conditions at pressures up to $\sim 5 \times 10^{-6} \mathrm{mbar}^{22}$ although at significantly higher pressures and temperatures their steady-state surface coverage was too low to detect. ${ }^{29}$ Here we show that the local geometries of both species when coadsorbed under these higher-pressure $\left(10^{-5} \mathrm{mbar}\right)$, higher-temperature $(450 \mathrm{~K})$ steady-state reaction conditions are similar to those found in the UHV experiments. We find, however, that the methoxy species no longer occupies the adatom-bridge sites of Fig. 1.

Guided by the previous results of Zhou et al. ${ }^{20,21}$ NAPXPS measurements of $\mathrm{C} 1 \mathrm{~s}$ and $\mathrm{O} 1 \mathrm{~s}$ emission were made under a range of different temperatures and total pressures

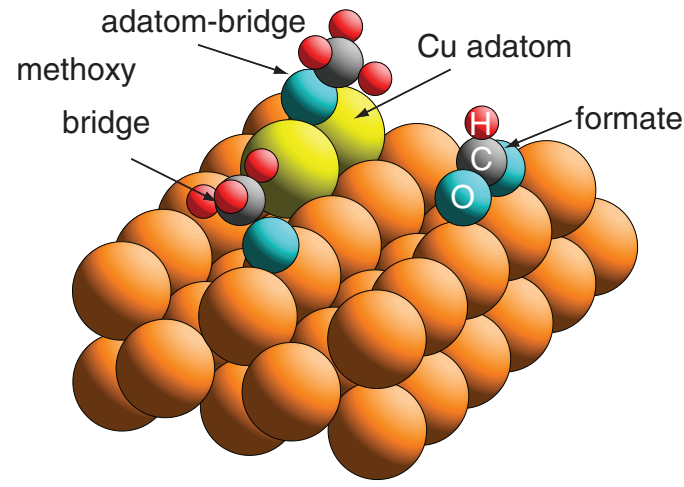

FIG. 1. (Color online) Schematic diagram showing the local adsorption sites occupied by the methoxy and formate species on $\mathrm{Cu}(110)$ under UHV conditions. The methoxy species forms an ordered $(5 \times 2)$ structure with equal occupation of the two different bridge sites.

of the reactant gases, monitoring the mass spectrum to identify the lowest temperatures corresponding to significant formaldehyde production. Lower temperatures reduce the amplitude of thermal vibrations of the surface atoms and thus minimize the impact of the Debye-Waller attenuation of the PhD modulations. All NAP-XPS and PhD data presented here were obtained under conditions corresponding to constant rate of production of formaldehyde; specifically, a steady-state increase in the intensity of the peaks in the mass spectra at $29 \mathrm{amu}$ and $30 \mathrm{amu}$ (with contributions from both methanol and formaldehyde), relative to that the peak at $31 \mathrm{amu}$ (only methanol), was observed. XPS data recorded under these reaction conditions at $450 \mathrm{~K}$, and a pressure of $10^{-5} \mathrm{mbar}$, are shown in Fig. 2. The three surface species that we might expect to see on the surface under reaction conditions are methoxy, formate, and atomic oxygen. Previously reported O 1s (and C

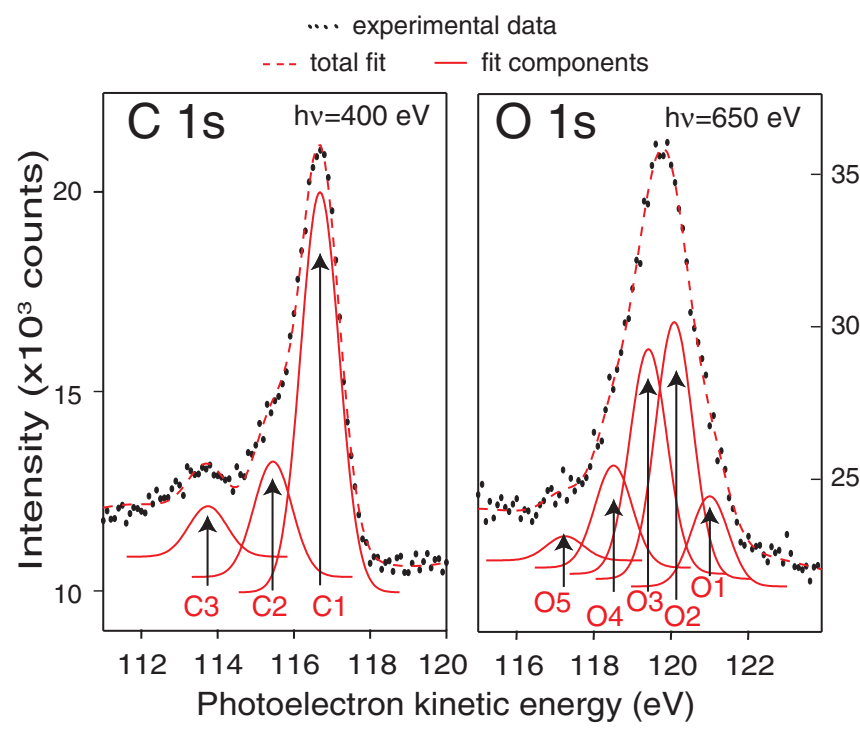

FIG. 2. (Color online) $\mathrm{C} 1 \mathrm{~s}$ and $\mathrm{O}$ 1s SXP spectra recorded from $\mathrm{Cu}(110)$ at $450 \mathrm{~K}$ with a total pressure of $10^{-5}$ mbar under steadystate reaction of methanol and oxygen to produce formaldehyde. The different spectral components corresponding to specific surface species are described in the text. 
1s) chemical shifts for these species vary significantly, ${ }^{22}$ but a consistent fit to a much larger set of spectra, obtained at other temperatures and pressures and showing varying relative contributions from different components, was achieved using a fixed set of chemical shift values. Specifically, O 1s chemical shifts of the methoxy and formate species relative to the atomic $O$ peak were found to be $1.00 \mathrm{eV}$ and $1.65 \mathrm{eV}$, respectively, with similar peak widths in the range of $0.85 \mathrm{eV}$ to $1.14 \mathrm{eV}$. Correct peak identification was aided by the fact that the $\mathrm{C} 1 \mathrm{~s}$ chemical shift between the methoxy and formate species is $2.3-2.5 \mathrm{eV},{ }^{22}$ much larger than the value for $\mathrm{O} 1 \mathrm{~s}$. An additional constraint in the consistent simultaneous fitting of both $\mathrm{C} 1 \mathrm{~s}$ and $\mathrm{O} 1 \mathrm{~s}$ spectra is the known $\mathrm{C}: \mathrm{O}$ stoichiometry of the methoxy and formate species (1:1 and 1:2, respectively). Following Günther et al., ${ }^{22}$ we therefore assign the three peaks in the $\mathrm{C} 1 \mathrm{~s}$ spectra to $\mathrm{CH}_{\mathrm{x}}$ (C1), $\mathrm{CH}_{3} \mathrm{O}(\mathrm{C} 2)$, and $\mathrm{HCOO}(\mathrm{C} 3)$. The $\mathrm{O} 1, \mathrm{O} 2$, and $\mathrm{O} 3$ peaks are assigned to $\mathrm{O}, \mathrm{CH}_{3} \mathrm{O}$, and $\mathrm{HCOO}$, respectively, but the origin of the $\mathrm{O} 4$ and $\mathrm{O} 5$ peaks is unclear. It is possible that these peaks are associated with molecular water and/or methanol that are transiently present on the surface in the presence of the gas phase. The energy dependence of these peaks showed no modulations above the noise level that would be ascribed to $\mathrm{PhD}$, indicating that they do not correspond to species adopting single high-symmetry sites close to the copper surface.

Figure 3 shows the $\mathrm{PhD}$ spectra recorded from the methoxy and formate components of the $\mathrm{O} 1 \mathrm{~s}$ spectra under the same conditions as the XPS spectra of Fig. 2. Equivalent data obtained from these same species prepared and measured under UHV conditions are also shown for comparison. Both

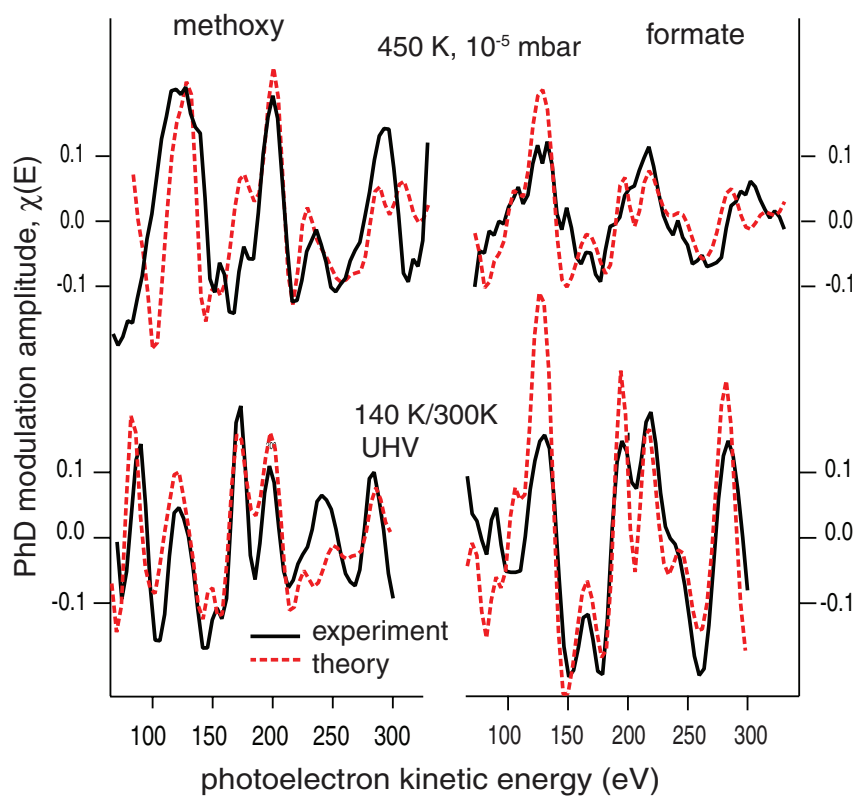

FIG. 3. (Color online) $\mathrm{O} 1 \mathrm{~s} \mathrm{PhD}$ spectra recorded in normal emission from the methoxy $\left(\mathrm{CH}_{3} \mathrm{O}\right)$ and formate $(\mathrm{HCOO})$ species on $\mathrm{Cu}(110)$. The upper spectra were recorded under steady-state methanol oxidation reaction conditions at $450 \mathrm{~K}$ and a pressure of $10^{-5}$ mbar. The lower spectra were recorded in the earlier studies under UHV at $140 \mathrm{~K}$ following reaction at $300 \mathrm{~K}$ (Refs. 25 and 28). The structural models associated with the theoretical simulations are described in the text. spectra from the formate species are dominated by a single long-period modulation, characteristic of normal emission from an atom in a near-atop site, when the scattering is dominated by this nearest neighbor that is located at a favorable $\sim 180^{\circ}$ scattering angle. Under reaction conditions, the modulations are weaker, and the fine structure of the UHV measurement is lost in the higher noise level; both effects can be largely attributed to the influence of increased vibrational amplitudes at the higher temperature at which the $\mathrm{PhD}$ spectra were recorded. The local adsorption geometry for the formate species is essentially identical under the two different conditions; the results of multiple scattering simulations, shown in Fig. 3, correspond to the same local geometries but with $\mathrm{Cu}-\mathrm{O}$ bondlengths of $1.90 \pm 0.03 \AA$ and $1.88 \pm 0.05 \AA$ for the UHV and reaction conditions, respectively. While the two methoxy $\mathrm{PhD}$ spectra also show somewhat similar underlying modulation envelopes, there are more significant differences in relative intensities and energies of the different peaks. Under UHV conditions, the methoxy $\mathrm{O}$ atom occupies bridging sites on the surface; therefore, normal emission does not correspond to a favored nearest-neighbor $180^{\circ}$ backscattering geometry. As a result, these $\mathrm{PhD}$ spectra do not show a single dominant underlying periodicity. In order to understand the differences between the spectra from the UHV and reaction conditions, multiple scattering simulations for a range of trial structures were performed using the same methods as those of the previous UHV study. ${ }^{25}$ Figure 3 shows the best fit to the new experimental data achieved in this way. Minor adjustments to the bond lengths and surface relaxations in the UHV two-site model (Fig. 1, which gave a high $R$-factor value of 0.79 ) failed to produce significant improvement in the fit between experiment and theory. Calculations for alternative sites investigated in the UHV study (shown in Ref. 30) give even worse agreement; however, removing the methoxy from adatom-bridge sites in the UHV two-site model produced the significant improvement shown in Fig. 3 with a much lower $R$ factor of 0.44 . In this model, the $\mathrm{Cu}(110)$ surface is essentially bulk terminated (with only $0.05 \AA$ outward surface relaxation), and the methoxy $\mathrm{O}$ atom occupies short-bridge sites with a $\mathrm{Cu}-\mathrm{O}$ bondlength of $1.89 \pm 0.08 \AA$, essentially identical to the value at this site in the UHV preparation. The two-site model is associated with the ordered $(5 \times 2)$ phase that occurs under UHV preparation conditions, methanol being deposited on a partially oxygenated surface at $140 \mathrm{~K}$ followed by heating to $\sim 300 \mathrm{~K}$. The $\mathrm{PhD}$ data in this earlier study were obtained after recooling to $140 \mathrm{~K}$ to avoid the rapid desorption and dissociation found to occur at $300 \mathrm{~K}$ in the presence of the incident photon beam. ${ }^{30}$ The constant arrival and desorption of methoxy under reaction conditions in the present measurements evidently allows $\mathrm{PhD}$ measurements to be made of surface methoxy at much higher temperatures. Some difference in the local structure under reaction conditions might not be surprising, not only because the long-range ordered phase is unlikely to occur, but also because of the dynamic nature of the steady-state reaction condition. The trapping and releasing of $\mathrm{Cu}$ adatoms by the atomic oxygen reactant species and associated higher mobility of $\mathrm{Cu}$ surface atoms (enhanced by the elevated temperature) might be expected to lead to a poor availability of nearestneighbor adatom pairs for methoxy adsorption, despite the 
fact that such sites are calculated to be energetically slightly favored for adsorption. ${ }^{25}$

This demonstration of the successful application of the $\mathrm{PhD}$ technique to gas pressures well above UHV conditions allows one to gain entirely new structural information on the geometry of surface reaction intermediates through in situ studies under reaction conditions. Note that the pressure range of $10^{-5}$ mbar used here was the one previously found to correspond to reasonable coverage of the reaction intermediates on the surface during the reaction. At this pressure, the capability of a current NAP-XPS instrument is not fully exploited, but there seems no reason to believe that the technique cannot be applied to the full (higher) pressure range of such instruments. Significant challenges to this wider exploitation do remain. In particular, instrumental modification to permit $\mathrm{PhD}$ data collection at a range of emission directions would allow a larger data set to be collected thus enabling more complete structure determinations. $\mathrm{PhD}$ spectra in a single direction can lead to ambiguities in structural interpretation, although this problem is largely eliminated if the results of a related complete study under UHV conditions are available, as in the present case. The attenuating effect of atomic vibrations on $\mathrm{PhD}$, and on all other diffraction techniques, could also prevent the application of the method to much higher temperatures, yet there remains considerable scope for further exploitation of the method.

\section{ACKNOWLEDGMENTS}

The authors thank the staff of BESSY for their support of the experiments and the BESSY facility for the award of the necessary beamtime.
*Present address: School of Chemistry, University of Witwatersrand, P.O. Wits, Johannesburg, 2050, South Africa.

${ }^{\dagger}$ Corresponding author: d.p.woodruff@warwick.ac.uk

${ }^{1}$ R. P. Eischens and W. A. Pliskin, Adv. Catal. 10, 1 (1958).

${ }^{2}$ N. Sheppard and J. W. Ward, J. Catal. 15, 50 (1969).

${ }^{3}$ F. M. Hoffmann, Surf. Sci. Rep. 3, 107 (1983).

${ }^{4}$ C. T. Herbschleb, S. C. Bobaru, and J. W. M. Frenken, Catal. Today 154, 61 (2010).

${ }^{5}$ R. W. Joyner, M. W. Roberts, and K. Yates, Surf. Sci. 87, 501 (1979).

${ }^{6}$ D. F. Ogletree, H. Bluhm, G. Lebedev, C. S. Fadley, Z. Hussain, and M. Salmeron, Rev. Sci. Instrum. 73, 3872 (2002).

${ }^{7}$ A. Knop-Gericke, E. Kleimenov, M. Hävecker, R. Blume, D. Teschner, S. Zafeiratos, R. Schlögl, V. A. Bukhtiyarov, V. V. Kaichev, I. P. Prosvirin, A. I. Nizovskii, H. Bluhm, A. Barinov, P. Dudin, and M. Kiskinova, Adv. Catal. 52, 213 (2009).

${ }^{8}$ H. Bluhm, M. Hävecker, A. Knop-Gericke, M. Kiskinova, R. Schlögl, and M. Salmeron, MRS Bull. 32, 1022 (2007).

${ }^{9}$ M. Salmeron and R. Schlögl, Surf. Sci. Rep. 63, 169 (2008).

${ }^{10}$ C. Escudero and M. Salmeron, Surf. Sci. 607, 2 (2013).

${ }^{11}$ A. Kolmakov, D. A. Dikin, L. J. Cote, J. Huang, M. K. Abyaneh, M. Amati, L. Gregoratti, S. Günther, and M. Kiskinova, Nature Nanotech. 6, 651 (2011).

${ }^{12}$ M. Behrens, F. Studt, I. Kasatkin, S. Kühl, M. Hävecker, F. AbildPedersen, S. Zander, F. Girgsdies, P. Kurr, B.-L. Kniep, M. Tovar, R. W. Fischer, J. K. Nørskov, and R. Schlögl, Science 336, 893 (2012).

${ }^{13}$ Y. B. He, M. Knapp, E. Lundgren, and H. Over, J. Phys. Chem. B 109, 21825 (2005).

${ }^{14}$ S. Ferrer, M. D. Ackermann, and E. Lundgren, MRS Bull. 32, 1010 (2007)
${ }^{15}$ D. P. Woodruff and A. M. Bradshaw, Rep. Prog. Phys. 57, 1029 (1994).

${ }^{16}$ D. P. Woodruff, Surf. Sci. Rep. 62, 1 (2007).

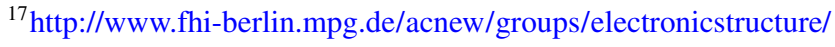
pages/beamline.html.

${ }^{18}$ I. E. Wachs and R. J. Madix, J. Catal. 53, 208 (1978).

${ }^{19}$ M. Bowker and R. J. Madix, Surf. Sci. 95, 190 (1980).

${ }^{20}$ L. Zhou, S. Günther, and R. Imbihl, J. Catal. 230, 166 (2005).

${ }^{21}$ L. Zhou, S. Günther, and R. Imbihl, J. Catal. 232, 295 (2005).

${ }^{22}$ S. Günther, L. Zhou, M. Hävecker, A. Knop-Gericke, E. Kleimenov, R. Schlögl, and R. J. Imbihl, J. Chem. Phys. 125, 114709 (2006).

${ }^{23}$ B. A. Sexton, A. E. Hughes, and N. R. Avery, Surf. Sci. 155, 366 (1986).

${ }^{24}$ P. Singnurkar, I. Bako, H. P. Koch, E. Dermirci, A. Winkler, and R. J. Schennach, J. Phys. Chem. C 112, 14034 (2008).

${ }^{25}$ M. K. Bradley, D. Kreikemeyer Lorenzo, W. Unterberger, D. A. Duncan, T. J. Lerotholi, J. Robinson, and D. P. Woodruff, Phys. Rev. Lett. 105, 086101 (2010).

${ }^{26}$ M. D. Crapper, C. E. Riley, and D. P. Woodruff, Surf. Sci. 184, 121 (1987).

${ }^{27}$ D. P. Woodruff, C. F. McConville, A. L. D. Kilcoyne, Th. Lindner, J. Somers, M. Surman, G. Paolucci, and A. M. Bradshaw, Surf. Sci. 201, 228 (1988).

${ }^{28}$ D. Kreikemeyer-Lorenzo, W. Unterberger, D. A. Duncan, M. K. Bradley, T. J. Lerotholi, J. Robinson, and D. P. Woodruff, Phys. Rev. Lett. 107, 046102 (2011).

${ }^{29}$ H. Bluhm, M. Hävecker, A. Knop-Gericke, E. Kleimenov, R. Schlögl, D. Teschner, V. I. Bukhtiyarov, D. F. Ogletree, and M. Salmeron, J. Phys. Chem. B 108, 14340 (2004).

${ }^{30}$ D. Kreikemeyer-Lorenzo, M. K. Bradley, W. Unterberger, D. A. Duncan, T. J. Lerotholi, J. Robinson, and D. P. Woodruff, Surf. Sci. 605, 193 (2011). 